\title{
Desde el Boletín de la Sociedad Chilena de Obstetricia y Ginecología a la Revista Chilena de Obstetricia y Ginecología: 80 años al servicio de la salud de la mujer chilena
}

El 15 de octubre de 1935 se funda la Sociedad Chilena de Obstetricia y Ginecología (SOCHOG), siendo su primer Presidente el Dr. Carlos Monckeberg B. (1935-1937). Simultáneamente, dadas las necesidades de compartir las experiencias clínicas de la especialidad y en especial beneficio de la salud de la mujer y del niño, nace en octubre de ese mismo año, el Boletín de la Sociedad Chilena de Obstetricia y Ginecología.

En 1935, Chile tenía una tasa de mortalidad fetal de 48,7/1000 nacidos vivos, mortalidad neonatal de 116/1000 nacidos vivos, mortalidad infantil de $251 / 1000$ nacidos vivos (1) y de mortalidad materna que superaba a $325 / 100.000$ nacidos vivos. Estas cifras representaban la dura realidad de la salud de la mujer chilena, que era un reflejo de la pobreza del país y que golpeaba cruelmente a las familias. Esta realidad era la que debía enfrentar los obstetras y ginecológos de la época, cuando las mujeres carecían aún de métodos anticonceptivos y debían recurrir frecuentemente al aborto para controlar su fecundidad.

Es así que la mortalidad materna por complicaciones del aborto en 1963 -previo a la incorporación de los métodos anticonceptivos- la mortalidad materna por aborto era de 106/100.000 nacidos vivos, correspondiendo al $38,8 \%$ de la mortalidad materna global (273/100.000 nacidos vivos) (2).

Lo anterior contrasta con lo que sucede con esos indicadores en la actualidad. Es así que las últimas cifras correspondiente al año 2012 entregadas por Instituto Nacional de Estadísticas son: mortalidad fetal de $8,5 / 1000$ nacidos vivos, mortalidad neonatal de 5,4/1000 nacidos vivos, mortalidad infantil de 7,4/1000 nacidos vivos (3) y mortalidad materna de 17,2/100.000 nacidos vivos (4). Las actuales cifras son producto del desarrollo social, educacional, económico y sanitario del país, a lo que ha contribuido nuestro órgano de difusión otorgando una plataforma para que los gineco-obstetras nacionales den a conocer sus experiencias clínicas y aportar conocimiento para la formación de los alumnos de pre y posgrado.

La biblioteca de la SOCHOG conserva prácticamente todos los volúmenes del Boletín Chileno de Obstetricia y Ginecología y de Revista Chilena de Obstetricia y Ginecología, los que están a disposición de la comunidad médica para consulta. Produce una enorme nostalgia cuando vemos que la Revista Chilena de Obstetricia y Ginecología tuvo presencia en Medline desde 1961 (5) hasta 1995 (6), con un total de 1.614 artículos publicados en forma continua y anteriormente como el Boletín de la Sociedad Chilena de Obstetricia y Ginecología desde 1946 (7) hasta 1960 (8), con 337 artículos. Ustedes pueden acceder a medline (http://www. ncbi.nlm.nih.gov/pubmed) y en su motor de búsqueda escribir rev chil obstet ginecol o bol chil obstet ginecol, y luego buscar en nuestra biblioteca los títulos seleccionados.

Navegando por el Boletín de la Sociedad Chilena de Obstetricia y Ginecología podrán encontrar materias que si bien son parte de la historia de la medicina chilena, era una realidad que a diario debían enfrentar nuestros maestros, solo con su experiencia y recursos terapéuticos muy limitados. Es fascinante encontrar artículos referente a la tuberculosis, que hacía estragos en la fertilidad de la mujer (9), y era una causa frecuente de mortalidad materna (10), como también la infección por B. perfringens importante causa de muerte materna pos aborto (11), las primeras experiencias en anestesia obstétrica (12); el manejo médico de la eclampsia (13), y varias publicaciones en 1952 referente a las experiencias de los Servicios Obstétricos en relación a las indicaciones de cesáreas, complicaciones pos 
cesárea y dehiscencia de la cicatriz, etc. En fin son muchos los artículos que muestran la historia de la obstetricia y la ginecología que escribieron nuestros maestros, cuando nuestra especialidad, y especialmente la obstetricia era un arte: jhonor a ellos!

En la actualidad la experiencia de nuestros maestros ha sido reemplazada por la medicina basada en la evidencia y las revistas biomédicas dan a conocer las experiencias acumuladas de las investigaciones a través de metaanálisis y revisiones sistemáticas, que permiten el mejor manejo de las pacientes. De ahí que es importante que las revistas biomédicas estén en buscadores internacionales que las seleccionan de acuerdo a una serie de variables que certifican su calidad científica. Nuestra Revista tiene como principal buscador la plataforma electrónica Scielo (Scientific Library Online) (http://www.scielo.org), proyecto de carácter regional, iniciativa de FAPESP (Fundación de Apoyo a la Investigación del Estado de São Paulo) y de BIREME (Centro Latinoamericano y del Caribe de Información en Ciencias de la Salud). En Chile, la plataforma Scielo está coordinada por la Comisión Nacional de Investigación Científica y Tecnológica (CONICYT) y es parte de su política de fomento a la investigación y difusión de sus resultados. Nuestra Revista fue incorporada en el año 2002 a la plataforma Scielo Chile (http:// www.scielo.cl), acumulando interrumpidamente
81 números correspondientes a 14 volúmenes (volumen 67 al 80).

En el año 2008, lamentablemente, por falta de financiamiento, el número 6 del volumen 73, fue el último número impreso, pasando solamente a difusión on-line en la plataforma Scielo y en el sitio de la Sociedad Chilena de Obstetricia y Ginecología (http://www.revistasochog.cl). Esto permitió una mejor difusión de la Revista convirtiéndose en un órgano hispanoamericano de difusión en la especialidad de la Obstetricia y Ginecología, siendo nuestros principales contribuyentes grupos de España, Venezuela, Colombia y México. Agradecemos a nuestros colegas de Hispanoamérica por compartir sus experiencias con nosotros.

Finalmente, deseo agradecer a los 15 editores jefes del Boletín de la Sociedad Chilena de Obstetricia y Ginecología y de la Revista Chilena de Obstetricia y Ginecología, lamentablemente, muchos de ellos ya no están con nosotros, quienes por su dedicación al cuidado de la salud de la mujer y amor a la especialidad, dedicaron muchas horas de trabajo y sacrificio personal a la continuidad y calidad de nuestro órgano de difusión, en 80 años de ininterrumpida difusión (Tabla I).

Dr. Enrique Donoso S. Editor Jefe.

Revista Chilena de Obstetricia y Ginecología.

Tabla I

EDITORES JEFES DEL BOLETÍN DE LA SOCIEDAD CHILENA DE OBSTETRICIA Y GINECOLOGÍA Y DE LA REVISTA CHILENA DE OBSTETRICIA Y GINECOLOGÍA, 1935-2015

\begin{tabular}{cll}
\hline Periodo & \multicolumn{1}{c}{ Editores Jefes } & \multicolumn{1}{c}{ Revista } \\
\hline $1935-1940$ & Víctor M. Gacitúa G. & Boletín de la Sociedad Chilena de Obstetricia y Ginecología \\
$1941-1945$ & Héctor Cruz M. & Boletín de la Sociedad Chilena de Obstetricia y Ginecología \\
$1946-1947$ & Onofre Avendaño P. & Boletín de la Sociedad Chilena de Obstetricia y Ginecología \\
$1948-1949$ & Rogelio Rodríguez B. & Boletín de la Sociedad Chilena de Obstetricia y Ginecología \\
$1950-1951$ & Luis Tisné B. & Boletín de la Sociedad Chilena de Obstetricia y Ginecología \\
$1952-1953$ & Fernando Rodríguez S. & Boletín de la Sociedad Chilena de Obstetricia y Ginecología \\
$1954-1955$ & Héctor Cabrera S. & Boletín de la Sociedad Chilena de Obstetricia y Ginecología \\
$1956-1959$ & Amalia Ernst M. y Eduardo Valenzuela. & Boletín de la Sociedad Chilena de Obstetricia y Ginecología \\
$1960-1960$ & Víctor M. Gacitúa G. & Boletín de la Sociedad Chilena de Obstetricia y Ginecología \\
$1961-1966$ & Gildo Zambra C. & Revista Chilena de Obstetricia y Ginecología \\
$1967-1977$ & Héctor Cruz M. & Revista Chilena de Obstetricia y Ginecología \\
$1978-1988$ & Onofre Avendaño P. & Revista Chilena de Obstetricia y Ginecología \\
$1989-2003$ & Mario Herrera M. & Revista Chilena de Obstetricia y Ginecología \\
$2004-2015$ & Enrique Donoso S. & Revista Chilena de Obstetricia y Ginecología \\
\hline
\end{tabular}




\section{REFERENCIAS}

1. Tendencia de la natalidad, mortalidad general, infantil y neonatal en Chile desde el año 1850 a la fecha. Águila A, Muñoz H. Rev Med Chile 1997;125:123645.

2. Armijo R, Monreal T. Epidemiología del aborto inducido en Santiago. Rev Chil Obstet Ginecol 1964;29:3342.

3. Instituto Nacional de Estadísticas de Chile. Anuario de Estadísticas Vitales 2012.

4. Tras el cumplimiento del $5^{\circ}$ Objetivo del Milenio: mortalidad materna en Chile, 2012 (Editorial). Donoso E. Rev Chil Obstet Ginecol 2015;80(1):7-11.

5. Cruz H, Fischer O, Guzmán A, Lillo F. [Choriocarcino$\mathrm{ma}$, with special reference to the diagnosis]. Rev Chil Obstet Ginecol 1961;26(1):2-20.

6. Aguilar GU, Barrero RP, Benavides AM, Dabancens AO, Smirnow MS. [Exceptional variety of endometrial cancer]. Rev Chil Obstet Ginecol 1995;60(3):219-21.
7. Vaccaro H, Paredes L, Aguilera VE. Bol Soc Chil Obstet Ginecol 1946;11(8):213-24.

8. Villavicencio $G$, de la Fuente R, Villablanca E. [Hepatic cirrhosis and the gravido-puerperal state]. Bol Soc Chil Obstet Ginecol 1960;25: 499-507.

9. Wood J, Elgueta H. [Experiences in the university gynecologic clinic with streptomycin therapy of tuberculosis of the uterine adnexa]. Bol Soc Chil Obstet Ginecol 1951;16(1):30-41.

10. Ugarte JM. [Maternal mortality in Chile]. Bol Soc Chil Obstet Ginecol 1952;17(7):178-84.

11. Villavicencio G. [General aspects of puerperal septico-toxemia caused by Bacillus perfringens]. Bol Soc Chil Obstet Ginecol 1955;20(5):113-24.

12. Keymer $\mathrm{E}$, Cereceda $\mathrm{H}$. [Experience with 50 cases of spinal anesthesia with dimecaine in obstetric interventions]. Bol Soc Chil Obstet Ginecol 1953;18(8):21821.

13. Puga JA, Varas $O$ [Treatment of convulsive eclampsia with infusion of sodium pentothal, preliminary communication]. Bol Soc Chil Obstet Ginecol 1955;20(67):155-8). 